Traditional Medicine and Modern Medicine

Vol. 1, No. 1 (2018) 11-13

(C) Institutes of Integrative Medicine, Fudan University and

World Century Publishing Corporation

DOI: $10.1142 / \mathrm{S} 257590001820001 \mathrm{X}$

\title{
Global situation and WHO strategy on traditional medicine
}

\author{
Qi Zhang \\ World Health Organization, Geneva, Switzerland \\ zhangqi@who.int
}

Received 7 November 2017; Accepted 20 December 2017; Published 2 February 2018

Health systems around the world are experiencing increased levels of chronic illness, population aging and escalating health care costs. Patients and health care providers alike are demanding that health care services should be revitalized with a stronger emphasis on individualized, person-centred care. This includes expanding access to traditional, complementary and integrative medicine (TCI) products, practices and practitioners, in particular, in primary health care (PHC).

Traditional medicine (TM) is often seen as more accessible, more affordable, and more acceptable to local populations and can therefore be a tool to help in achieving the universal health coverage (UHC). ${ }^{1}$ There has been a continuing demand for, and popular use of, TCI worldwide. In some developing countries, native healers remain the sole or main health providers for millions of people living in rural areas. For instance, the ratio of traditional health practitioners to citizens in Africa is 1:500, whereas the ratio of medical doctors to citizens is $1: 40,000 .^{2}$ In the Lao People's Democratic Republic, $80 \%$ of the population live in rural areas, with each village being serviced by one or two traditional health practitioners. ${ }^{3}$ Over 100 million Europeans are currently users of TCI, with one-fifth being regular users; a similar proportion chooses healthcare that includes TCI. ${ }^{4}$ According to a national survey in China, practitioners of traditional Chinese medicine received 907 million visits from patients in 2009 , which accounts for $18 \%$ of all medical visits to surveyed institutions. Further, the number of traditional Chinese medicine inpatients was 13.6 million, or $16 \%$ of the total in all hospitals surveyed. ${ }^{5}$

More and more countries are coming to accept the contribution that TCI can make to the health and well-being of individuals and the comprehensiveness of their health care systems. In the period 1999 to 2012, the number of member states of WHO with national policies covering traditional medicine (TM) has increased significantly. This includes countries better regulating herbal medicines, or creating national research institutes to study TCI. ${ }^{6}$ In Africa, the number of national regulatory frame works increased from 1 in 1999/ 2000 to 28 in $2010 .^{7}$ Across the Atlantic, the Ministry of Health in Brazil has developed a national policy on integrative and complementary practices,${ }^{8}$ while in the Eastern Mediterranean region, five member states reported having regulations specifically for TCI practitioners. ${ }^{6}$ Member states in the South-East Asia Region are now pursuing a harmonized approach to education, practice, research, documentation and regulation of $\mathrm{TM}^{6}$; in Japan, $84 \%$ of Japanese physicians use Kampō (Japanese TM) in daily practice. ${ }^{9}$ In Switzerland, certain complementary therapies have been reinstated into the basic health insurance scheme available to all Swiss citizens. ${ }^{10}$

Recently, during the implementation of WHO TM Strategy 2014-2023, the World Health Assembly resolutions on TM (WHA67.18, adopted in 2014) and on strengthening integrated, people-centred health services (WHA69.24, adopted in 2016), we have been observing the increased number of Member States establishing national policy/strategy/workplan towards the integration of traditional and complementary medicine (T\&CM) into national health systems. In a few countries, certain types of TM have been completely integrated into the health care system, including China, the Democratic People's Republic of Korea (North Korea), the Republic of Korea (South Korea), India and Vietnam. In many other countries, T\&CM is partially integrated into the national health system, while in some countries, there is no integration so far. The integrative approaches of $\mathrm{T} \& \mathrm{CM}$ and conventional medicine are contributing in innovative ways of drug discovery and clinical research. The 
success of artemisinin, a new drug from Chinese herbal medicines by Youyou Tu, the winner of Nobel Prize in 2015, is an example of using the integrative approach of TM and conventional medicine in scientific research.

Despite the significant advances and progress in the past, the regulation of TCI products, practices and practitioners is not occurring at an equal pace. ${ }^{6}$ Member states report that faster progress is being made in the regulation of herbal medicines, while that for TCI practices and practitioners is lagging. Of concern is that the safety, quality and efficacy of TCI services cannot be assured if there is no appropriate regulation of practices and practitioners. This situation presents a serious challenge for many member states, where a lack of knowledge and experience exists regarding the formulation of national policy, leading to weak or absent regulation and a lack of proper integration of T\&CM services into the health service delivery system. Responding to the needs and challenges identified by the member states and building on the work done under the WHO TM Strategy 2002-2005, ${ }^{11}$ the updated strategy for the period 2014-2023 devotes more attention than its predecessor to health services and systems, including TCI products, practices and practitioners. The three key objectives of the updated strategy are summarized as follows.

Objective 1 is to build the knowledge base for active management of T\&CM through appropriate national policies. The first strategic step is to understand and recognize the role and potential of TCI and to build country profile in each country. The second strategic step recommends that member states strengthen knowledge generation, collaboration and sustainable use of TCI resources, including intellectual and natural resources. Objective 2 is to strengthen quality assurance, safety, proper use and effectiveness of T\&CM by regulating $\mathrm{T} \& \mathrm{CM}$ products, practices and practitioners. The first strategic element is emphasizing on the monitoring and implementation of established regulations of TM products and ensuring that information on quality and safety is shared internationally so that products are used appropriately in a global market and the appropriate harmonization and convergence of regulations and standards are also supported. The second strategic direction is to recognize and develop TCI practice and practitioner regulations for education and training, skills development, services and therapies. Objective 3 is to promote UHC by integrating $\mathrm{T} \& \mathrm{CM}$ services into health care service delivery and self-health care. A first step is to capitalize on the potential contribution of TCI to improve health services and health outcomes. Next is to ensure that consumers of TCI can make informed choices about selfhealthcare since self-selection of TCI products accounts for a large part of the TCI market. ${ }^{6}$

The WHA67.18 resolution on TM urges member states to adapt, adopt and implement the WHO TM Strategy
2014-2023 as a basis for national T\&CM programs or workplans and to report to WHO on progress in implementing the strategy. The WHA69.24 resolution on strengthening integrated, people-centred health services urges member states to integrate $\mathrm{T} \& \mathrm{CM}$ into health services while assuring safety, quality and effectiveness of health services and taking into account a holistic approach to health. ${ }^{12}$ In the implementation of the WHO TM strategy and the WHA resolutions, several lines of work have been initiated in WHO based on WHO core functions, primarily how to integrate T\&CM into health systems particularly in PHC, standards and norms development, and research and capacity building.

In the line of leadership, WHO is first working on supporting countries in the appropriate integration of $\mathrm{T} \& \mathrm{CM}$ into national health systems particularly primary health services, including the work on criteria for assessment of the integration and on identifying good models in the countries. WHO is also working on essential knowledge, skills and training requirements for PHC practitioners, and on self-care and mhealth including consumer education and engagement. Tai ji health in traditional Chinese medicine and Yoga in Indian TM are good examples in this area. Second, working on quality assurance and improvement of TCI practices. Third, strengthening network, such as the network of international regulatory cooperation for herbal medicines (IRCH), WHO collaborating centres for TCI, WHO expert advisory panel for TCI and the non-governmental organizations in the area of TCI in official relations with WHO.

In the line of standards and norms development, three categories of work are currently going on. One is the essential standards, including a series of terminologies and classifications in TCI. Terminologies are covering different modalities of TCI including Ayurveda, traditional Chinese medicine, Thai TM and Unani medicine. The example of classification is inclusion of TM into WHO ICD-11. The second category of standards is a series of benchmarks for training and a series of benchmarks for practice in TCI. The third category is guidelines and monographs on herbal medicines. Recently, the WHO guidelines on marker substances from herbal origin for quality control of herbal medicines and WHO guidelines on good herbal processing practices (GHPP) for herbal medicines were approved by the WHO Expert Committee. Some other guidelines or technical documents are also in the developing process.

In the line of research and knowledge and ethical and evidence-based policy, WHO is working with partners to jointly establish the TCI clinical research and knowledge databases and platforms, and supporting countries in the implementation of the WHO TM strategy. In the line of building institutional capacity, WHO continues in organizing the inter-regional training workshops on different topics of TCI for capacity building of governmental officials 
responsible for TCI in WHO Member States. WHO is also developing the training materials or tools to support the capacity building. In the line of monitoring and assessing the situation of TCI, WHO is monitoring the implementation of the TM strategy 2014-2023, conducting regular global surveys for building up a database as repository of Member States situation and assessing the trends of TCI.

The health goal in the UN Sustainable Development Goals (SDGs) is to ensure healthy lives and promote wellbeing for all at all ages. TCI has great potential to contribute to the UHC and SDGs particularly through strengthening its role in PHC. The goals of WHO TM Strategy 2014-2023 are to support member states in harnessing the potential contributions of TCI to health, wellness, people-centred healthcare and UHC, while also promoting safe and effective use of TCI through the regulation, research and integration of $\mathrm{T} \& \mathrm{CM}$ products, practices and practitioners into the national healthsystem as appropriate.

\section{References}

1. Qi Zhang. Health for all. In: Traditional and Complementary Medicine in Primary Health Care. Chap. 12. York: Centre for Global Health Histories, The University of York, 2015:93-94.

2. Abdullahi AA. Trends and challenges of traditional medicine in Africa. Afr J Tradit Complement Altern Med 2011;8:115.

3. Lao Ministry of Health and World Health Organization. Health Service Delivery Profile, Lao PDR, 2012 (compiled incollaboration between WHO and Ministry of Health, Lao PDR, 2012). Available at: http://www.wpro.who.int/health_ services/service_delivery_profile_laopdr.pdf.

4. European Information Centre for Complementary \& Alternative Medicine. Available at: http://www.eiccam.eu/home.php? $\mathrm{il}=1 \& \mathrm{l}=$ eng.

5. State Administration of Traditional Chinese Medicine, Report of a survey on T\&CM basic situation in 2009. 2011;6.

6. WHO Traditional Medicine Strategy 2014-2023 Geneva: World Health Organization, 2013:15-56.

7. World Health Organization, Progress report on decade of traditional medicine in the Africa region. Brazzaville: WHO African Region (AFRO), 2011.

8. Availabel at: http://bvsms.saude.gov.br/bvs/publicacoes/pnpic_ access_expansion_initiative.pdf.

9. Moschik EC, Mercada C, Yoshino T, Matsuura K, Watanabe K. Usage and attitudes of physicians in Japan concerning traditional Japanese medicine (kampo medicine): A descriptive evaluation of a representative questionnaire-based survey. Evid Based Complement Alternat Med 2012;2012:13.

10. Swiss Confederation, Five CAM methods eligible for reimbursement under specific conditions for aprovisional period of six years, 2011. Availabel at: www.bag.admin.ch/aktuell/ 00718/01220/index.html?lang=de\&msg-id=37173.

11. WHO Traditional Medicine Strategy 2002-2005. Geneva: World Health Organization, 2002.

12. World Health Organization, WHO Governing Body Documentation Official Records. Availabel at: http://apps.who.int/ gb/or/. 